\section{Brazilian Journal of Chemical Engineering}

ISSN 0104-6632

Printed in Brazil

www.abeq.org.br/bjche

Vol. 33, No. 01, pp. 155 - 168, January - March, 2016

dx.doi.org/10.1590/0104-6632.20160331s00001267

\title{
ADVANCED CONTROL OF A COMPLEX CHEMICAL PROCESS
}

\author{
Roxana Both ${ }^{1}$, Eva-Henrietta Dulf ${ }^{1^{*}}$ and Ana-Maria Cormos ${ }^{2}$ \\ ${ }^{1}$ Technical University of Cluj Napoca, Memorandumului St., No. 28, RO - 400014, Cluj-Napoca, Romania. \\ ${ }^{2}$ Babes-Bolyai University, Faculty of Chemistry and Chemical Engineering, \\ Arany Janos St., No. 11, RO-400028, Cluj-Napoca, Romania. \\ Phone: + 40-264-401821; Fax: + 40-0264-401220 \\ E-mail: Eva.Dulf@aut.utcluj.ro; http://aut.utcluj.ro
}

(Submitted: July 25, 2011 ; Revised: February 12, 2015 ; Accepted: February 21, 2015)

\begin{abstract}
Three phase catalytic hydrogenation reactors are important reactors with complex behavior due to the interaction among gas, solid and liquid phases with the kinetic, mass and heat transfer mechanisms. A nonlinear distributed parameter model was developed based on mass and energy conservation principles. It consists of balance equations for the gas and liquid phases, so that a system of partial differential equations is generated. Because detailed nonlinear mathematical models are not suitable for use in controller design, a simple linear mathematical model of the process, which describes its most important properties, was determined. Both developed mathematical models were validated using plant data. The control strategies proposed in this paper are a multivariable Smith Predictor PID controller and multivariable Smith Predictor structure in which the primary controllers are derived based on Internal Model Control. Set-point tracking and disturbance rejection tests are presented for both methods based on scenarios implemented in Matlab/SIMULINK.

Keywords: Multivariable Smith Predictor; Internal Model Control; Hydrogenation process; Dynamic mathematical model.
\end{abstract}

\section{INTRODUCTION}

The industrial scale 2-ethyl-hexenal hydrogenation process produces 2-ethyl-hexanol oxo-alcohol, used as raw material for dioctyl-phthalate (DOP) or 2ethylhexyl-phthalate (DEHP) production. DOP and DEHP represent around $50 \%$ of the global consumption in the production of poly(vinyl chloride) (PVC) (ICIS,2011;WRI,2009;SRI,2011).

Because the hydrogenation reaction is highly exothermic, the reactor output temperature is of great importance. Like most of chemical and petro-chemical plants, the 2-ethyl-hexenal hydrogenation process presents large time delays and is multivariable by nature. For multivariable time delay processes, the control solution will revolve around a multivariable Smith Predictor. A very important step for the multi- variable Smith Predictors is the decoupling of the process.

The present paper is structured in 8 parts. After the introduction, the second part describes the hydrogenation process and also details the actual control and the new proposed control system. The third part presents the developed dynamic mathematical models of the hydrogenation process: the nonlinear distributed parameter mathematical model and the linear simplified mathematical model. Both models are validated using plant data, acquired courtesy of S.C. Oltchim S.A., Ramnicu Valcea, Romania. In the control algorithm design procedure for multi-input multi-output (MIMO) systems, it is necessary a measure of interactions within MIMO systems, which can be provided by the Relative Gain Array (RGA) analysis presented in section four. Sections

*To whom correspondence should be addressed 
five and six present a multivariable PID controller design, respectively IMC control design. In section seven the comparative simulation results between the two control strategies are presented. The conclusions represent the last part of the paper.

\section{PROCESS DESCRIPTION}

The 2-ethyl-hexenal hydrogenation process is complex, which can take place both in liquid phase or gas phase in the presence of a catalyst and, besides the main product (2 ethyl-hexanol), can also form other side-products like: n-butanol or iso-butanol from secondary side-reactions. Technological solutions present both advantages and disadvantages. The gas phase 2-ethyl-hexenal hydrogenation reaction is characterized by high energy consumption and great dimensions of equipment, but has an important advantage: the possibility to regenerate the catalyst. In comparison to the gas phase hydrogenation reaction, the advantages of the liquid phase hydrogenation reaction of 2-ethyl-hexenal are: low energy consumption, small dimension of equipment, and small volumes of catalyst. However, the main drawback is the impossibility of catalyst regeneration. Industrial scale liquid phase hydrogenation is predominant in chemical industries and represents the main focus of the present work.

An important role in the hydrogenation reaction is played by the catalyst. For the present case study, a nickel catalyst deposited on silica support was chosen based on the BASF license. This type of catalyst for the 2-ethyl-hexenal hydrogenation reaction is also recommended in specific literature (Smelder, 1989).

Several researchers like Bozga (2001) and Cocker (2011) studied the hydrogenation reaction mechanism. Hence, the reaction mechanism stages for the present case study are (Bozga et al., 2001; Cocker, 2011): the transport of hydrogen from the gas phase to the gas-liquid interface; the transport of hydrogen from the gas-liquid interface to the liquid phase; the transport of reactants 2-ethyl-hexenal and hydrogen from the liquid phase to the liquid-solid interface; reactant adsorption to the active sites of the catalyst; the chemical reaction between adsorbed reactant molecules; product desorption from the active sites of the catalyst; the product transport from the catalyst surface to the liquid phase. The reaction pathway is expressed as follows (Collins et al., 2009):

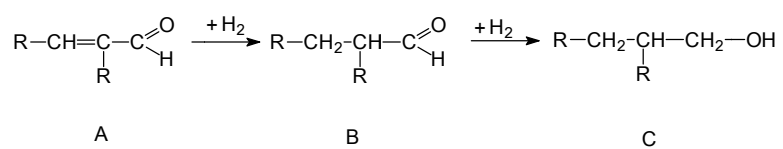

where: A is 2-ethyl-hexenal (reactant), B is 2-ethylhexanal (intermediate product) and $\mathrm{C}$ is 2-ethylhexanol (final product).

The main parameters that influence the two consecutive hydrogenation reactions (the intermediate product being 2-ethyl-hexanal) are: the pressure, temperature (input/output), reactor loading, reflux rate and purity of input reactants (hydrogen, 2-ethylhexenal). The temperature is a critical parameter due to the fact that the hydrogenation reaction is highly exothermic with a reaction heat of $25.27 \times 10^{3}$ $\mathrm{kcal} / \mathrm{kmol}$ (Smelder,1989). High temperatures favor side-reactions, but also an input temperature of the reactants between $90^{\circ} \mathrm{C}$ and $110^{\circ} \mathrm{C}$ is necessary in order to start the reaction. The maximum technological recommended temperature inside the reactor is in the range of $160{ }^{\circ} \mathrm{C}-180^{\circ} \mathrm{C}$, depending on the catalyst degree of activity. The solution chosen to maintain the temperature below the critical value is the dilution of the 2-ethyl-hexanal flow with the product itself (the reflux rate). A typical industrial process flow diagram of the 2-ethyl-hexenal hydrogenation process is presented in Figure 1.

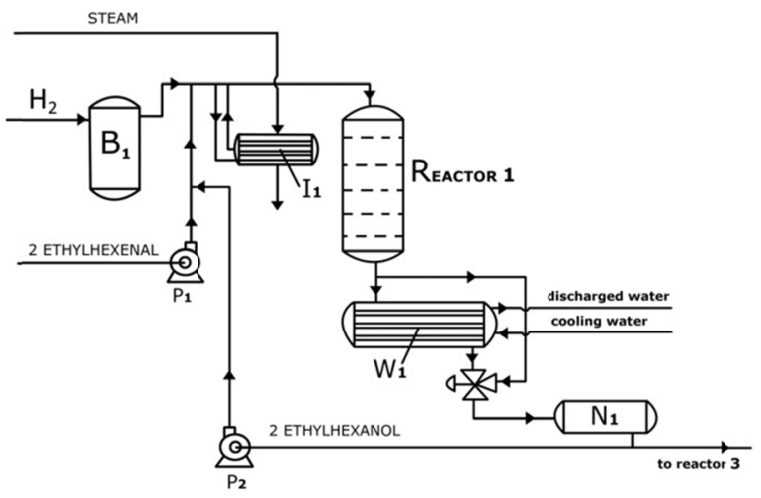

Figure 1: Process flow diagram of a typical 2-ethylhexenal hydrogenation process used in industrial plants.

As presented before, a parameter of great importance is the reactor loading. This influences the hydrogenation reaction itself and also the temperature. It was determined experimentally that a specific flow ratio between the 2-ethyl-hexenal flow and the recirculated 2-ethyl-hexanol flow must be maintained to carry out the reaction with good parameters and also to maintain the temperature in the recommended range. The catalyst degree of activity acts as a disturbance because it influences the hydrogenation reaction and implicitly the output temperature of the product. The necessary steps to counteract the effect of a decrease in the catalyst activity are: to change 
the flow ratio (the recirculated 2-ethyl-hexanol flow must be decreased) and also to increase the input temperature of the reactants to start the hydrogenation reaction.

At the present time, the hydrogenation reactor is operated using an open loop control system, so the main parameters like 2-ethyl-hexenal input flow rate, recirculated 2-ethyl-hexanol flow and input temperature of the reactants are maintained at the imposed values, without feedback control of output temperature or 2-ethyl-hexanol output concentration. This control solution is characterized by a main disadvantage: it is not fault-tolerant, being difficult to apply correction methods if one control loop fails. However, the hydrogenation reactor will operate in a satisfactory manner if the auxiliary control loops for flow control of the reactants and input temperature control of the reactants are working in good parameters.

At present an operator sets manually the reference values for the 2-ethyl-hexenal input flow rate, recirculated 2-ethyl-hexanol flow and input temperature of the reactants. The 2-ethyl-hexanol output concentration is measured periodically and, if it is not satisfactory, the reference values for the main input variables are adjusted by the accumulated expertise of the operator. In order to optimize the production, the authors propose a feedback control system using the existing infrastructure. The implementation costs are reduced to the costs of a temperature transducer used to measure the output temperature of the product.

In order to ensure safe and normal conditions of operation for the hydrogenation process there are two main goals of the control strategy. The first goal is to maintain the temperature at an acceptable value. This goal can be achieved by assuring a specific volumetric flow ratio between the 2-ethyl-hexenal and 2-ethyl-hexanol flow rates. The recirculated 2-ethyl-hexanol flow rate is considered to be the manipulated variable. The second goal is to ensure a high conversion of 2-ethyl-hexenal to 2-ethyl-hexanol. This goal can be reached by assuring the necessary input temperature of the reactants to start the reaction. The input temperature of the reactants must be increased as the catalyst degree of activity decreases in time. The input temperature of the reactants also has an important influence on the output temperature. Overall, the two considered control inputs are the 2 ethyl-hexanol flow rate and the input temperature of the reactants. The output temperature and product concentration are considered to be the measured outputs and the input hydrogen pressure $(P)$, and 2-ethyl-hexenal flow (Qenal) act as disturbances.

\section{MATHEMATICAL MODELS}

\section{Distributed Parameter Nonlinear Mathematical Model}

An accurate mathematical model is needed to evaluate the operational challenges and to understand the processes that occur inside the reactor and also to develop a more efficient control strategy. In the specific literature, a number of kinetic studies of the hydrogenation reaction are reported (Smelder, 1989; Niklasson, 1987, 1988). However, no mathematical model of the process is given.

Motivated by these reasons, in previous works (Both, 2013), a first principle based mathematical model was developed starting only from some equations describing the hydrogenation reaction kinetics. The developed model (Both, 2013), consisting of numerous partial differential equations and several algebraic ones, was implemented and validated using plant data acquired courtesy of S.C. Oltchim S.A. This model is useful in the design, optimization and operation of the chemical reactor and especially in control design and testing. The reaction rate equations, transport models, energy and mass balances were coupled and were solved with respect to time and space with algorithms suitable for partial differential equations (Attou et al., 1999; Burghardt et al., 1995, Iliuta et al., 1997).

The equations describing the nonlinear mathematical model used are (Both, 2013):

- The total mass balances for the gas and liquid phases are (Both, 2013):

$$
\begin{aligned}
& \frac{\partial F_{L}}{\partial t}=-v_{L} \frac{\partial F_{L}}{\partial z}+v_{L} \cdot S \cdot M_{H_{2}} \cdot a_{v} \cdot N_{H_{G}} \\
& \frac{\partial F_{G}}{\partial t}=-v_{G} \frac{\partial F_{G}}{\partial z}-v_{G} \cdot S \cdot M_{H_{2}} \cdot a_{v} \cdot N_{H_{G}}
\end{aligned}
$$

- The component mass balances for liquid and gas phases are (Both, 2013):

$$
\begin{aligned}
& \frac{\partial C_{H}^{L}}{\partial t}=-v_{L} \frac{\partial C_{H}^{L}}{\partial z}+a_{v} \cdot N_{H_{G}}-v p h \\
& \frac{\partial C_{H}^{G}}{\partial t}=-v_{L} \frac{\partial C_{H}^{G}}{\partial z}-a_{v} \cdot N_{H_{G}} \\
& \frac{\partial C_{\text {enal }}}{\partial t}=-v_{L} \frac{\partial C_{\text {enal }}}{\partial z}-r_{1} \cdot \rho_{\text {sol }}
\end{aligned}
$$




$$
\begin{aligned}
& \frac{\partial C_{\text {anal }}}{\partial t}=-v_{L} \frac{\partial C_{\text {anal }}}{\partial z}+\left(r_{1}-r_{2}\right) \cdot \rho_{\text {sol }} \\
& \frac{\partial C_{o c t}}{\partial t}=-v_{L} \frac{\partial C_{o c t}}{\partial z}+r_{2} \cdot \rho_{\text {sol }}
\end{aligned}
$$

- The heat balances for the liquid and gas phases are (Both, 2013):

$$
\begin{aligned}
& \frac{\partial T_{L}}{\partial t}=-v_{L} \frac{\partial T_{L}}{\partial z}-\sum \frac{\Delta_{R} H_{i} \cdot r_{i}}{c_{p L}} \\
& \frac{\partial T_{G}}{\partial t}=-v_{G} \frac{\partial T_{G}}{\partial z}-\sum \frac{\Delta_{R} H_{i} \cdot r_{i}}{c_{p G}}
\end{aligned}
$$

where $F_{L}$ and $F_{G}$ are the liquid and gas phase flow, respectively, $S$ is the cross-sectional area, $a_{v}$ is the specific gas-liquid contact area, $N_{H G}$ is the flux of hydrogen transferred from gaseous phase to liquid phase, $C_{\text {enal }}$ is the concentration of 2-ethyl-hexenal, $C_{a n a l}$ is the concentration of 2-ethyl-hexanal, $C_{o c t}$ is the concentration of 2-ethyl-hexanol, $C_{H}^{L}$ is the concentration of hydrogen in the liquid phase and $C_{H}^{G}$ is the concentration of hydrogen in the gaseous phase. $v p h$ is the transferred hydrogen flow through the liquid film, adjacent to the catalyst pellet, onto its surface (Bozga, 2001) and $r_{i}$ is the rate of surface reaction $i$ and $\Delta_{R} H_{i}$ is the reaction heat of reaction $i$.

- The reaction rates are described by (Smelder, 1989):

$$
r_{1}=\frac{k_{1} K_{\text {enal }} K_{H} C_{\text {enal }} C_{H_{2}}}{\left(1+K_{\text {enal }} C_{\text {enal }}+K_{\text {anal }} C_{\text {anal }}+\sqrt{K_{H} C_{H_{2}}}\right)^{3}}
$$

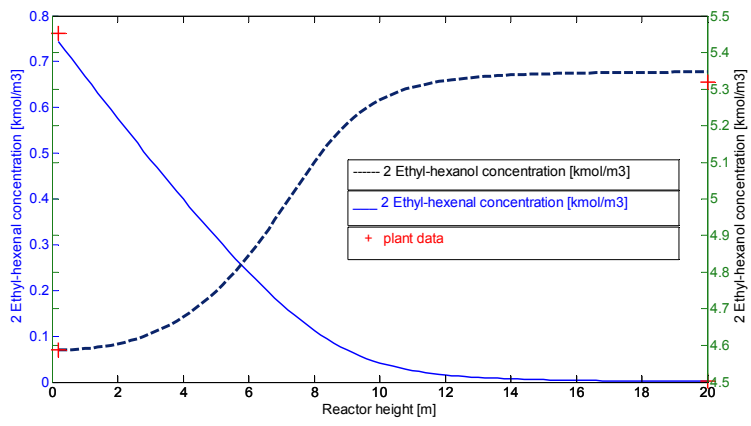

Figure 2: 2-ethyl-hexenal and 2-ethyl-hexanol concentration profile along the reactor height RUN 1.

$$
r_{2}=\frac{k_{2} K_{\text {anal }} K_{H} C_{\text {anal }} C_{H_{2}}}{\left(1+K_{\text {enal }} C_{\text {enal }}+K_{\text {anal }} C_{\text {anal }}+\sqrt{K_{H} C_{H_{2}}}\right)^{3}}
$$

where $K_{i}$ is the adsorption equilibrium constant for component $i$ ( $i$ : enal, anal, $\mathrm{H}), C_{i}$ concentration of component $i$ and $k_{i}$ rate constant of surface reaction $i,(i: 1,2)$.

The rate of reaction incorporating the catalyst deactivation can be obtained as follows:

$$
r_{i, d}=r_{i} a(t)
$$

where: $a$ is a fraction of active catalyst and $r_{i}$ is the reaction rate of species $i$.

For validation of the developed model a considerable number of trials were chosen to compare simulation results with plant data (NIST, 2011; Gas-

\begin{tabular}{|c|c|c|c|c|c|c|c|c|}
\hline \multirow[t]{2}{*}{ Run } & \multicolumn{2}{|c|}{$\begin{array}{c}\mathrm{H}_{2} \\
\text { concentration } \\
\left(\mathrm{kmol} / \mathrm{m}^{3}\right)\end{array}$} & \multicolumn{2}{|c|}{$\begin{array}{c}\text {-ethyl-hexenal } \\
\text { concentration } \\
\left(\mathrm{kmol} / \mathrm{m}^{3}\right)\end{array}$} & \multicolumn{2}{|c|}{$\begin{array}{c}\text { 2-ethyl-hexanol } \\
\text { concentration } \\
\left(\mathrm{kmol} / \mathrm{m}^{3}\right)\end{array}$} & \multicolumn{2}{|c|}{$\begin{array}{c}\text { Outlet liquid } \\
\text { temperature } \\
\text { (K) }\end{array}$} \\
\hline & sim & & & & & & & \\
\hline 1 & & & & & & & & \\
\hline 2 & 0.088 & 0.055 & 0.0022 & 0.0023 & & 5.31 & & 441.5 \\
\hline
\end{tabular}
par et al., 2010; Perry et al., 1999; Tobiensen et al., 2008, Silva et al., 2003) and the two most representative cases are presented in Table 1, which emphasizes the small differences between the plant data and simulated values.

Table 1: Data validation.

The accuracy of the distributed parameter nonlinear mathematical model of the process is highlighted by the evolutions of 2-ethyl-hexenal, 2-ethyl-hexanol concentrations and the temperature evolution along the reactor height, presented in Figure 2 and Figure 3.

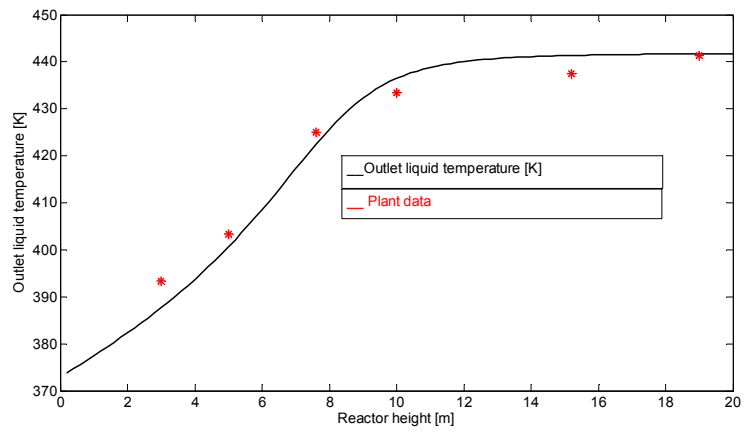

Figure 3: Outlet liquid temperature profile along the reactor height - RUN 1. 
Operational Mathematical Model

Due to the fact that nonlinear mathematical models are more accurate, but also too complex for efficient use in controller design, the authors propose another approach, to use a simple operational model of the process which describes its most important properties (Iancu et al., 2010; Simson et al., 2001).

The linear operational mathematical model was determined using experimental identification methods based on plant data, as a transfer function matrix of second order elements between the main input and output variables (Both, 2012): the 2-ethyl-hexanol recirculated flow (Qoct), the input temperature of the reactants (Tin), the output temperature of the product (Tout) and the output concentration of the product (Cout).

$H_{p}(s)=\left|\begin{array}{ll}H f_{11}(s) \cdot e^{-s \tau} q o & H f_{12}(s) \cdot e^{-s \tau_{t i}} \\ H f_{21}(s) \cdot e^{-s \tau_{q o c}} & H f_{22}(s) \cdot e^{-s \tau_{t i c}}\end{array}\right|$

where

$$
\begin{aligned}
& H f_{11}(s)=\frac{K_{q o}}{T_{q o 1} s^{2}+T_{q o 2} s+1} \\
& H f_{12}(s)=\frac{K_{t i}}{T_{t i 1} s^{2}+T_{t i 2} s+1} \\
& H f_{21}(s)=\frac{K_{q o c}}{T_{q o c 1} s^{2}+T_{q o c 2} s+1} \\
& H f_{22}(s)=\frac{K_{t i c}}{T_{t i c 1} s^{2}+T_{t i c 2} s+1}
\end{aligned}
$$

The input hydrogen pressure $(P)$ and 2-ethyl-hexenal flow (Qenal) act as disturbances. The transfer matrix describing the dependence between the output variables and the disturbances is:

$$
H_{\text {dist }}(s)=\left|\begin{array}{ll}
H f_{11 \text { dist }}(s) \cdot e^{-s \tau_{q e}} & H f_{12 \text { dist }}(s) \cdot e^{-s \tau} p \\
H f_{21 \text { dist }}(s) \cdot e^{-s \tau_{q e c}} & H f_{22 \text { dist }}(s) \cdot e^{-s \tau} p
\end{array}\right|
$$

where

$$
H f_{11 d i s t}(s)=\frac{K_{q e}}{T_{q e 1} s^{2}+T_{q e 2} s+1}
$$

$$
\begin{aligned}
& H f_{12 \text { dist }}(s)=\frac{K_{p}}{T_{p 1} s^{2}+T_{p 2} s+1} \\
& H f_{21 \text { dist }}(s)=\frac{K_{q e c}}{T_{q e c 1} s^{2}+T_{q e c 2} s+1} \\
& H f_{22 \text { dist }}(s)=\frac{K_{p c}}{T_{p 1 c} s^{2}+T_{p 2 c} s+1}
\end{aligned}
$$

Following the previous equations, the transfer matrix of the simplified hydrogenation reactor is:

$$
\left|\begin{array}{c}
\text { Tout } \\
\text { Coct }
\end{array}\right|=H_{p}(s) \cdot\left|\begin{array}{c}
\text { Qoct } \\
\text { Tin }
\end{array}\right|+H_{\text {dist }}(s) \cdot\left|\begin{array}{c}
\text { Qenal } \\
P
\end{array}\right|
$$

Using the operational mathematical model, the plant data and the simulation results of the nonlinear validated mathematical model, analysis of the dynamical behavior of the simplified model is done. Figure 4 and Figure 5 present the evolution of the output temperature (Tout) for a $+14 \%$ step variation of the 2-ethyl-hexenal input flow and for a step variation in the 2-ethyl-hexanol recirculated flow.

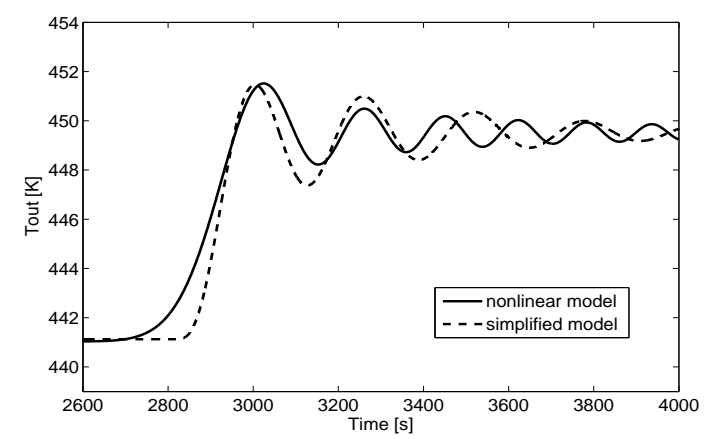

Figure 4: Outlet liquid temperature evolution: change in Qenal.

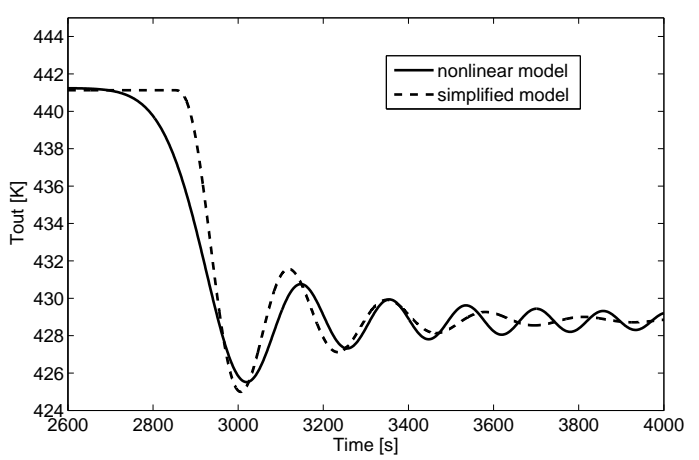

Figure 5: Outlet liquid temperature evolution: change in Qoct. 
The validation of the simplified linear mathematical model is based on the comparison between equation results and plant data. The presence of an acceptable error is emphasized in the previous figures.

\section{RELATIVE GAIN ARRAY (RGA) ANALYSIS}

Considerable research effort was dedicated to the development of control techniques for MIMO processes, but it still represents a difficult task in practical engineering. At the present time, there are two main directions of approach in control design for multivariable processes. The first approach supports the idea to design a centralized MIMO controller, which implies the use of algebraic decoupling methods or optimal control theory. However, the result is a complex controller, difficult to implement. Another approach is to design a decentralized controller, which attempts to control a fairly multivariable process by separating the control problem into several SISO (single - input, single - output) systems and then uses conventional control for each loop. The advantages of this approach are very clear: simple to design, easy to implement or tune (fewer parameters); hence it is more often used in practical applications. However, the main disadvantage is represented by the substandard closed loop performance due to interactions between loops. A mandatory step is to determine the appropriate loop configuration in order to obtain minimal interactions between loops. This is achieved by proper pairing of the manipulated and controlled variables. In the chemical industry the process complexity reaches up to several hundred control loops, making input - output pairing a difficult task. This task is also essential because it can lead to an unstable overall system, even if the individual loops are stable.

In 1996, Bristol (1966) introduced the Relative Gain Array technique to determine a measure of the process interactions, giving advice in solving the pairing problem. This technique considers only the steady-state gains of the process to determine the best pairing solution. Due to its simplicity, the RGA technique was widely used in different applications and still has the widest application in industry. The main disadvantage of this technique is represented by the lack of dynamic information of the process, which may sometimes lead to an incorrect loop pairing decision. A dynamic version of the RGA technique was developed by McAvoy (1983). The dynamic RGA (DRGA), in comparison with the RGA, uses the transfer function model instead of the steadystate gain matrix in order to consider the process dynamics, giving a more accurate interaction assessment. Xiong (2005) combined in his work the advantages of both RGA and DRGA by introducing the concept of an effective relative gain array (ERGA), which provides an accurate, detailed and easy to understand description of the dynamic interaction among individual loops without the need to specify the controller type.

In this study the RGA technique was used to determine the best control loop pairs and, at the end, the performances of the selected control loop pairs are studied. RGA is represented by a matrix with one column for each input variable and one row for each output variable. From this matrix one can easily compare the relative gains associated with each inputoutput variable pair and match the input and output variables that have the biggest effect on each other, while also minimizing undesired side effects. Standard analysis suggests that RGA elements corresponding to input-output pairings close to 1 should be preferred. Negative or large RGA elements are disadvantageous as they correspond to loops which may be nominally stable, but which become unstable if saturation occurs (e.g. (Bristol, 1966)).

The simplest approach was found to be the use of the developed linear mathematical model and and to compute RGA from the steady-state gain matrix. The steady-state gain matrix is thus obtained with its elements:

$\left|\begin{array}{l}\text { Tout } \\ \text { Coct }\end{array}\right|=\left|\begin{array}{ll}K_{q o} & K_{t i} \\ K_{\text {qoc }} & K_{\text {tic }}\end{array}\right| \cdot\left|\begin{array}{c}\text { Qoct } \\ \text { Tin }\end{array}\right|$

The RGA, for a non-singular square matrix $G$, is a square complex matrix defined as $\operatorname{RGA}(G)=G \times\left(G^{-1}\right)^{T}$, where $x$ denotes element-by element multiplication (Hadamard or Schur product).

For the obtained steady-state gain matrix $H_{p 0}$

$H_{p 0}=\frac{\operatorname{Qoct}}{\operatorname{Tin}}\left(\begin{array}{cc}-2.1 & -0.0049 \\ 1 & 0.011\end{array}\right)$

and

$R G A\left(H_{p 0}\right)=\frac{\operatorname{Qoct}}{\operatorname{Tin}}\left(\begin{array}{cc}\text { Tout } & \text { Coct } \\ 1.2692 & -0.2692 \\ -0.2692 & 1.2692\end{array}\right)$

From the $R G A$ matrix one can observe that there is a strong coupling between Qoct and Tout and Tin and Coct. 


\section{MULTIVARIABLE SMITH PREDICTOR PID CONTROLLER DESIGN}

The previously presented linear simplified operational mathematical model of the hydrogenation process consisting of a transfer matrix $\mathrm{Hp}$, which describes the influence of the control inputs $u$ on the controlled outputs $y$ (Tout, Cout), and a transfer matrix $\mathrm{H}_{\text {dist }}$, describing the effect of disturbances in the feed $z$ (the input 2-ethyl-hexenal flow Qenal, hydrogen pressure $P$ ) on the outputs $y$ is used in order to develop a multivariable PID controller.

$y=H_{p} \cdot u+D \cdot z$

Both matrices Hp and D are (2,2)-matrices.

$$
\begin{gathered}
H_{p}=\left|\begin{array}{cc}
\frac{K_{q o} \cdot e^{-s \tau_{q o}}}{T_{q o 1} s^{2}+T_{q o 2} s+1} & \frac{K_{t i} \cdot e^{-s \tau_{t i}}}{T_{t i 1} s^{2}+T_{t i 2} s+1} \\
\frac{K_{q o c} \cdot e^{-s \tau_{q o c}}}{T_{q o c 1} s^{2}+T_{q o c 2} s+1} & \frac{K_{t i c} \cdot e^{-s \tau_{t i c}}}{T_{t i c 1} s^{2}+T_{t i c 2} s+1}
\end{array}\right| . \\
D=\left|\begin{array}{ll}
\frac{K_{q e} \cdot e^{-s \tau_{q e}}}{T_{q e 1} s^{2}+T_{q e 2} s+1} & \frac{K_{p} \cdot e^{-s \tau_{p}}}{T_{p 1} s^{2}+T_{p 2} s+1} \\
\frac{K_{q e c} \cdot e^{-s \tau_{q e c}}}{T_{q e c 1} s^{2}+T_{q e c 2} s+1} & \frac{K_{p c} \cdot e^{-s \tau_{p c}}}{T_{p 1 c} s^{2}+T_{p 2 c} s+1}
\end{array}\right|
\end{gathered}
$$

The hydrogenation process is characterized by the presence of time delays. A typical approach to deal with time delay is the non-delayed output prediction (Melo, 2008; Wang, 2008). The non-delayed output may be estimated and the controller can be computed as for a process without delay. The most popular output predictor is the Smith Predictor, Figure 6.

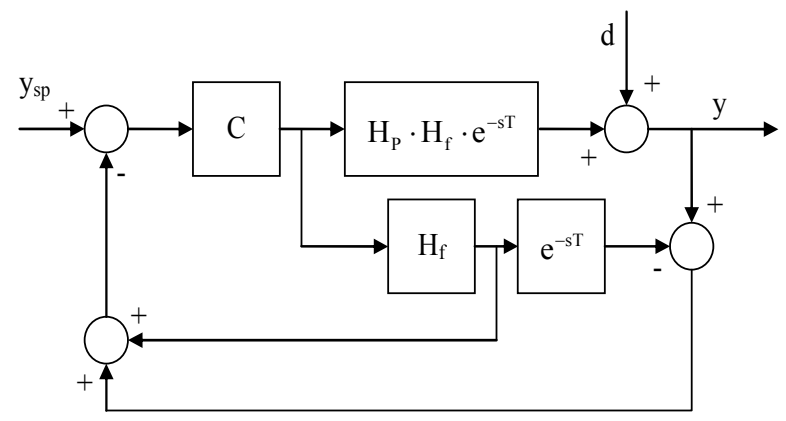

Figure 6: Smith predictor structure.
For the controller design the transfer matrix of the process without disturbance is used:

$$
\begin{aligned}
H_{p}(s) & =\left|\begin{array}{ll}
\frac{K_{q o} \cdot e^{-s \tau_{q o}}}{T_{q o 1} s^{2}+T_{q o 2} s+1} & \frac{K_{t i} \cdot e^{-s \tau_{t i}}}{T_{t i 1} s^{2}+T_{t i 2} s+1} \\
\frac{K_{q o c} \cdot e^{-s \tau_{q o c}}}{T_{q o c 1} s^{2}+T_{q o c 2} s+1} & \frac{K_{t i c} \cdot e^{-s \tau_{t i c}}}{T_{t i c 1} s^{2}+T_{t i c 2} s+1}
\end{array}\right| \cdot= \\
& =\left|\begin{array}{ll}
H f_{11}(s) \cdot e^{-s \tau_{q o}} & H f_{12}(s) \cdot e^{-s \tau_{t i}} \\
H f_{21}(s) \cdot e^{-s \tau_{q o c}} & H f_{22}(s) \cdot e^{-s \tau_{t i c}}
\end{array}\right|
\end{aligned}
$$

The desired multivariable controller matrix has the following form [34]:

$$
H_{R}(s)=\left|\begin{array}{ll}
H R_{11}(s) & H R_{12}(s) \\
H R_{21}(s) & H R_{22}(s)
\end{array}\right|
$$

where $H R_{11}, H R_{22}$ are designed for the direct control of the outputs and $H R_{12}, H R_{21}$ are designed to compensate the coupling effect.

Considering the large time delays of the plant the Smith Predictor structure is used, presented in Figure 7.

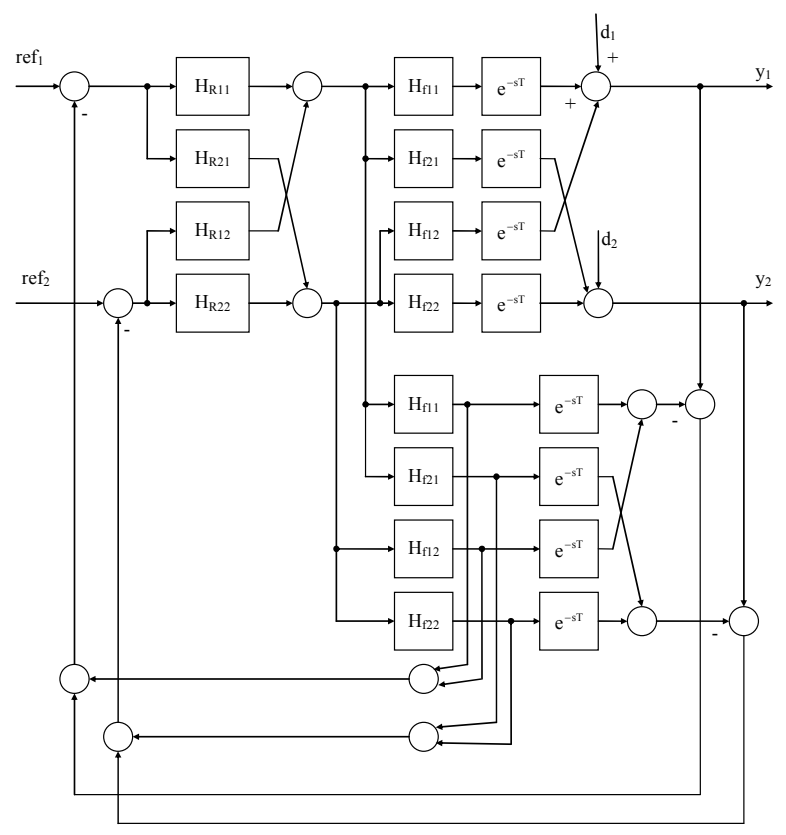

Figure 7: Closed loop control scheme using the Smith predictor structure.

All the controllers are computed using the imposed phase margin design method, for $\gamma_{k}{ }^{*}=60^{\circ}$.

The obtained controllers are: 


$$
\begin{aligned}
& H R_{11}(s)=1.3259\left(1+\frac{1}{102.3018 \cdot s}\right) \\
& H R_{12}(s)=2.7733\left(1+\frac{1}{106.6667 \cdot s}\right) \\
& H R_{21}(s)=595.6621\left(1+\frac{1}{90.4977 \cdot s}\right) ; \\
& H R_{22}(s)=223.8721\left(1+\frac{1}{119.0476 \cdot s}\right)
\end{aligned}
$$

\section{INTERNAL MODEL CONTROL DESIGN}

As presented before, the most popular structure for time delay compensation is the Smith Predictor structure. A very important step in the multivariable Smith Predictor structure is the decoupling of the process. A modified multivariable Smith Predictor structure was proposed by Wang, Zou and Zhang (Wang et al., 2000). In this case the decoupling matrix is computed in frequency domain. More recently the Internal Model Control (IMC) method was used in order to compute the decoupling matrix (Wang et al., 2002). Chen et al. (2011) used the IMC method only as an intermediary step in order to compute the final controller - a matrix of PI controllers. The method used in this paper is based on the approach proposed by Pop et al. (2011) in which the IMC controllers are used as final controllers.

The model of the process is assumed to be equal to the process transfer function matrix $H p$, presented before:

$$
H_{m}(s)=\left|\begin{array}{ll}
H f_{11 m}(s) \cdot e^{-s \tau_{q o}} & H f_{12 m}(s) \cdot e^{-s \tau_{t i}} \\
H f_{21 m}(s) \cdot e^{-s \tau_{q o c}} & H f_{22 m}(s) \cdot e^{-s \tau_{t i c}}
\end{array}\right|
$$

The next steps in order to design the IMC controllers are: a) Determine the pseudo-inverse matrix; b) Determine the decoupled process; c) Approximate the elements on the first diagonal of the steady-state decoupled process matrix; d) Design the IMC controllers.

The method used to decouple the multivariable system is the pseudo-inverse (Skogestad, 2011) of the steady state gain matrix:

$$
H_{m}(0)=\left|\begin{array}{ll}
H f_{11 m 0} & H f_{12 m 0} \\
H f_{21 m 0} & H f_{22 m 0}
\end{array}\right|
$$

The pseudo-inverse is computed based on the following equation (Skogestad, 2011):
$H_{m}^{\#}=H_{m}(0)^{H} \cdot\left(H_{m}(0) \cdot H_{m}(0)^{H}\right)^{-1}$

where $H_{m}^{\#}$ is the pseudo-inverse which will act as a pre-compensator matrix.

The decoupled multivariable process is done by:

$H_{D}(s)=H_{m}(s) \cdot H_{m}^{\#}=\left|\begin{array}{ll}H f_{11 d} & H f_{12 d} \\ H f_{21 d} & H f_{22 d}\end{array}\right|$

The matrix (36) represents the steady-state decoupled process, in which all elements are weighted sums of the original transfer functions $H f_{i j} \cdot e^{-s \tau}$. Due to the static decoupling, the steady-state matrix $H_{D}(s)=0$ will be equal to the unit matrix. In this way all the elements which are not on the first diagonal will be equal to zero.

The next step is to approximate the elements on the first diagonal of the matrix $H_{D}(s)$ with simple transfer functions:

$H f_{\text {iid }}(s) \approx H f_{\text {iid }}{ }^{*}(s)$

The elements on the first diagonal, approximated by the transfer function given in (37), will be used for controller design.

The approximation of each element $H f_{i i d}{ }^{*}(s)$ can be obtained by using genetic algorithms or graphical identification methods (Chen et al., 2011).

The next step is to design the IMC controller as follows (Chen et al., 2011):

$$
H R_{I M C i}(s)=H f_{i i d-i n v}^{*}(s) \cdot f(s)
$$

where $f(s)$ is the IMC filter and $H f_{i i d-i n v}{ }^{*}(s)$ represents the invertible part of $\mathrm{Hf}_{\text {iid }}{ }^{*}(s)$. The IMC filter is computed as:

$$
f(s)=\frac{1}{\left(\lambda_{i} s+1\right)^{n}}
$$

where $\lambda_{i}$ is the time constant of the filter associated with each output and $n$ is chosen such that the final IMC controller is proper. The parameters of the IMC controller will be tuned in accordance with the imposed performance values of overshoot and settling time. Figure 8 and Figure 9 show the closed loop response of $H f_{11 d}$ and $H f_{22 d}$ considering different values of the IMC filter, $\lambda=5,10,15,20$. Analyzing the simulation results in terms of settling time and 
overshoot, the final IMC controllers for the case study results in the following transfer function:

$$
\begin{aligned}
& H R_{I M C 1}=\frac{1000 s^{2}+55.85 s+1}{400 s^{2}+40 s+1} \text { and } \\
& H R_{I M C 2}=\frac{20001 s^{2}+87.2 s+1}{100 s^{2}+20 s+1}
\end{aligned}
$$

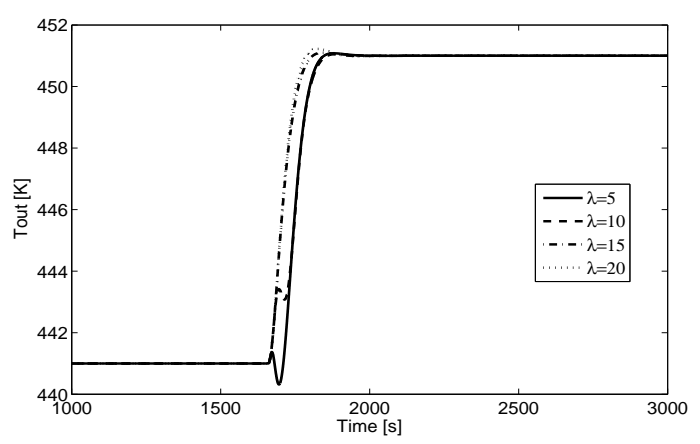

Figure 8: Tuning of the controller $\mathrm{IMC}_{1}$.



Figure 9: Tuning of the controller $\mathrm{IMC}_{2}$.

Figure 10 presents the closed loop control scheme using delay compensator and IMC controller. The Moore-Penrose pseudo-inverse $H_{m}^{\#}$ is used as a precompensator for the process $H_{p}(s)$, the model $H_{m}(s)$ and the fast model $\tilde{H}_{m}(s)$ (the process model without the time delays). $H_{D}{ }^{*}(s)$ is the transfer function matrix of all elements $H f_{i i d}{ }^{*}(s)$ and $\tilde{H}_{D}^{*}(s)$ is the transfer function matrix $H_{D}{ }^{*}(s)$ without the time delay.

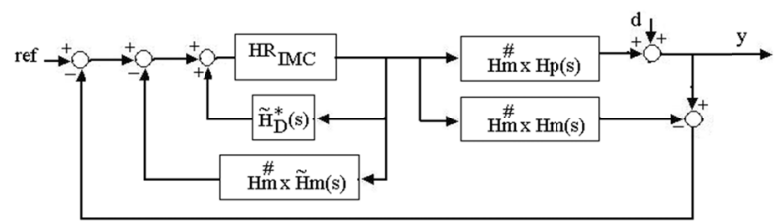

Figure 10: Closed loop control scheme using IMC controllers.

\section{SIMULATION RESULTS}

For analysis purposes both control structures were implemented in Matlab/SIMULINK (Matlab User Guide, 2008). The simulation scenarios are presented in comparison for the two control strategies in order to conclude the results. The two main objectives of the scenarios are the set-point tracking analysis and disturbance rejection analysis. Due to the fact that, for both control strategies, the controllers were designed based on the linear model of the process, it is necessary to test the robustness of the control strategies considering the variation of the model parameters. This analysis is performed in the third simulation scenario.

The first simulation scenario is focused on testing the set-point tracking performances of the designed control strategies.

To this end a reference step variation of $10 \mathrm{~K}$ for the output temperature of the product at $t=10 \mathrm{~s}$ is considered. Figure 11 presents the evolution of the output temperature while Figure 12 shows the influence of the reference step variation for the output temperature on the 2-ethyl-hexanol concentration.

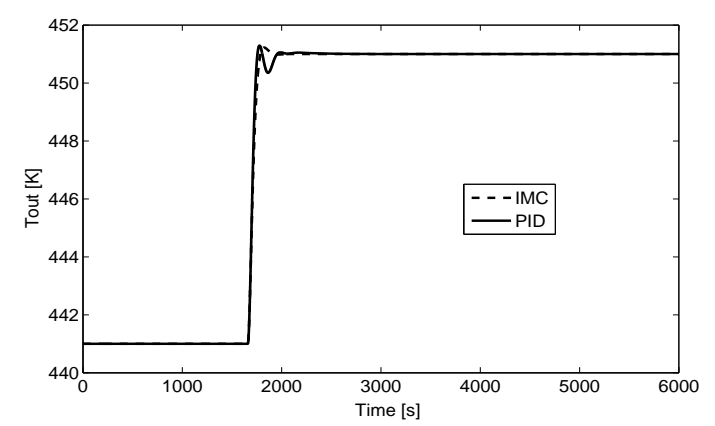

Figure 11: Output temperature evolution for a step variation on input 1.

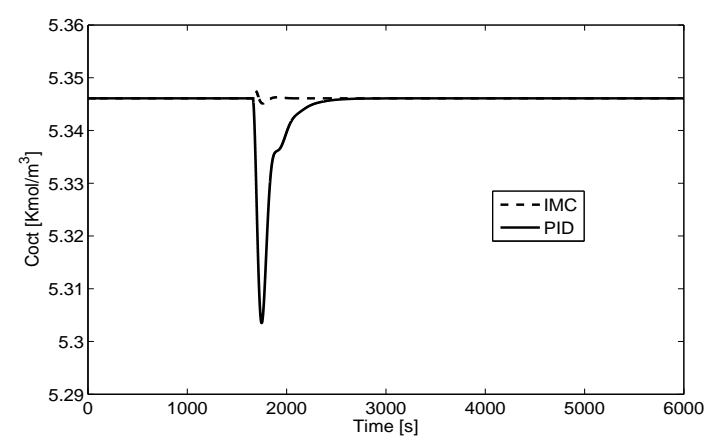

Figure 12: 2-Ethyl-hexanol concentration evolution for a step variation on input 1 .

For the same analysis a reference step variation of $0.25\left[\mathrm{kmol} / \mathrm{m}^{3}\right]$ is considered for the 2-ethyl-hexanol 
output concentration. The evolution of the 2-ethylhexanol concentration is presented in Figure 13. and the influence of the reference step variation on the output temperature is detailed in Figure 14.

To reach the second objective of the section, the second simulation scenario is focused on the disturbance rejection analysis of the designed control strategies. Figure 15 and Figure 16 present the effects of a $0.25\left[\mathrm{kmol} / \mathrm{m}^{3}\right]$ disturbance in 2-ethyl-hexenal flow on the two considered outputs.

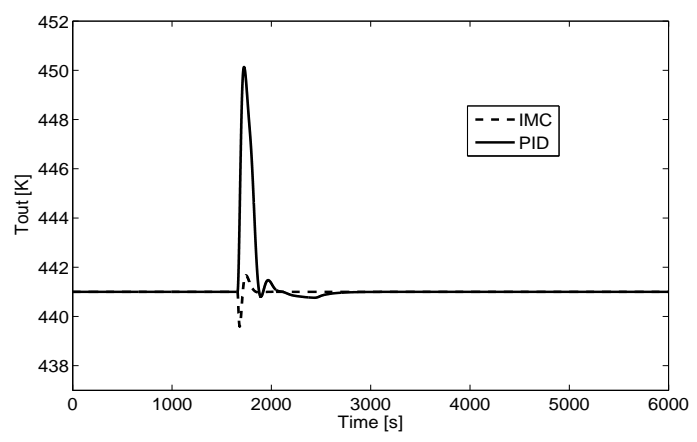

Figure 13: Output temperature evolution for a step variation on input 2 .

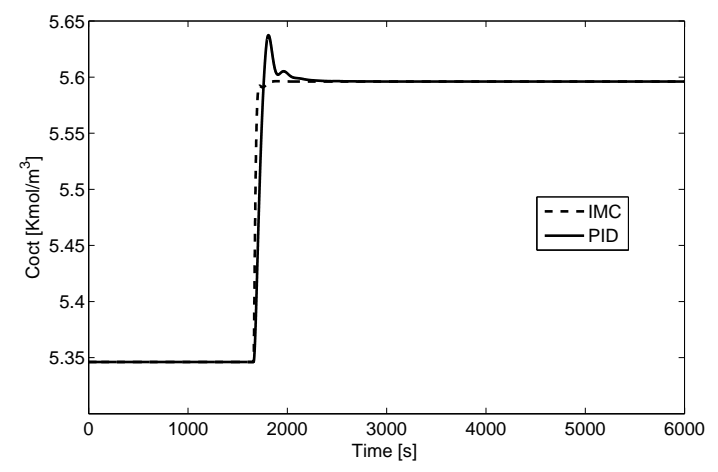

Figure 14: 2-Ethyl-hexanol concentration evolution for a step variation on input 2 .

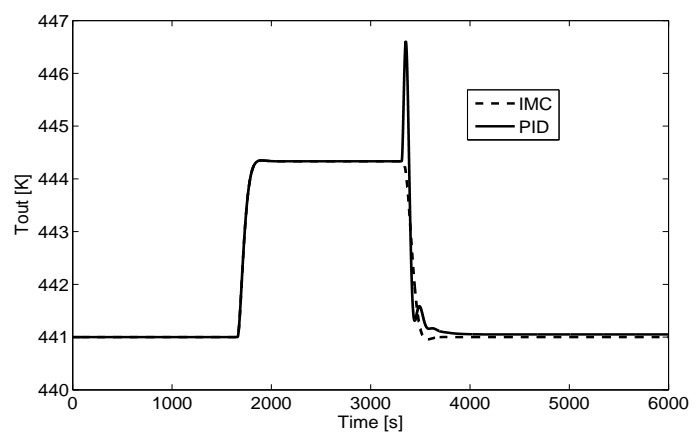

Figure 15: Output temperature evolution for a disturbance in 2-ethyl-hexenal flow.

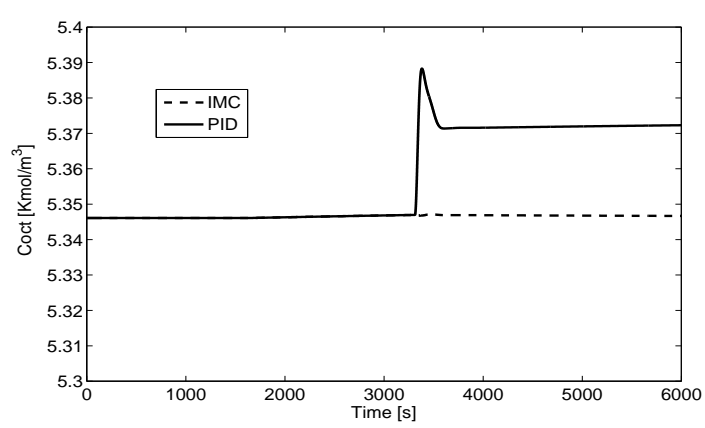

Figure 16: 2-Ethyl-hexanol concentration evolution for a disturbance in 2-ethyl-hexenal flow.

From all the above figures it can be concluded that the IMC control strategy presents better performances in comparison with the MIMO PID control. Another advantage of the method is that it is more straightforward and easy to design. The minor disadvantage is the Lag-Lead form of the controllers opposite to the classical PID forms.

The last objective of this section is to test the robustness of the designed control strategies. To this end, the following figures present the evolution of the output temperature and 2-ethyl-hexanol concentration considering a reference step variation for both variables and also the variation of the uncertain parameters of the linear plant.

The closed loop simulations using the multivariable Smith Predictor PI controller under nominal parameter values (solid black line), as well as the simulations considering the variation of the uncertain parameters of the linear plant (solid grey lines), for a reference step variation of the output temperature are presented in Figure 17 and Figure 18. The uncertain parameters considered are the time constants of the process as well as the gain parameter.

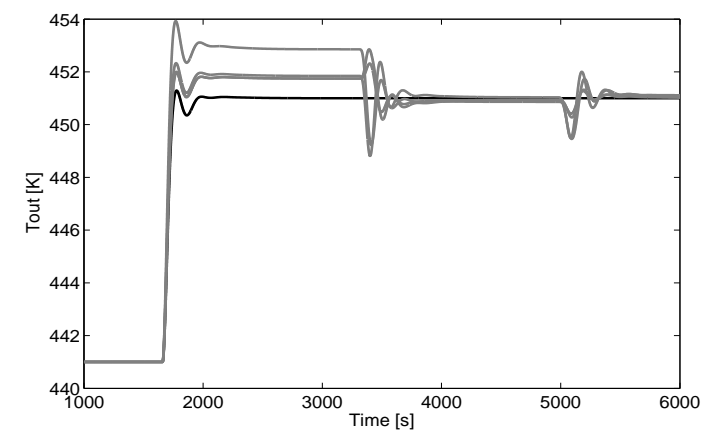

Figure 17: Output temperature evolution for a step variation on input 1 - nominal case vs. uncertain case (PID). 


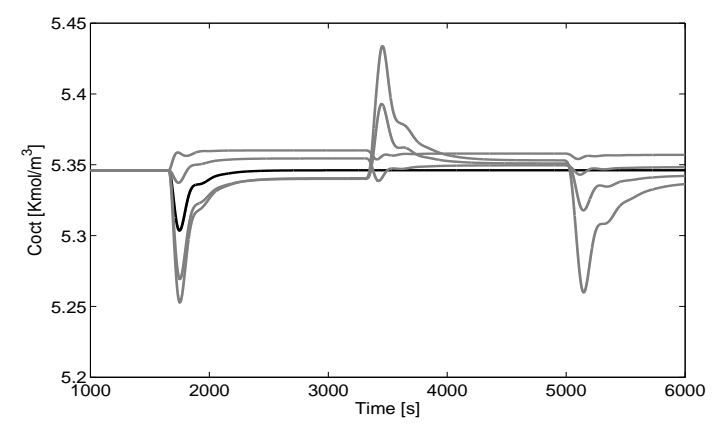

Figure 18: 2-Ethyl-hexanol concentration evolution for a step variation on input 1 - nominal case vs. uncertain case (PID).

The closed loop simulations using the multivariable Smith Predictor PI controller under nominal parameter values (solid black line), as well as the simulations considering the variation of the uncertain parameters of the linear plant (solid grey lines), for a reference step variation of the 2-ethyl-hexanol concentration are presented in Figure 19 and Figure 20.

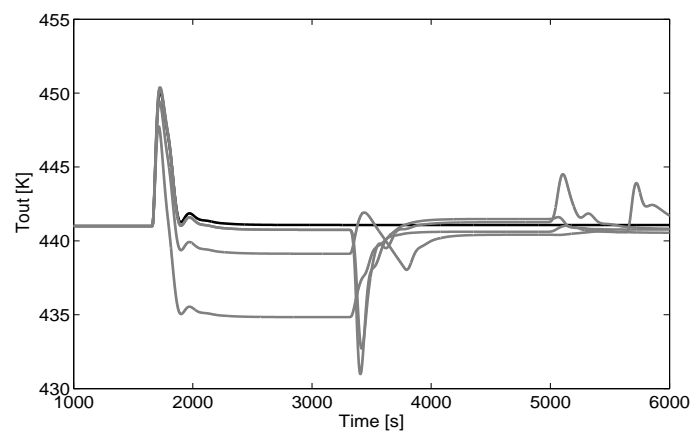

Figure 19: Output temperature evolution for a step variation on input $2-$ nominal case vs. uncertain case (PID).

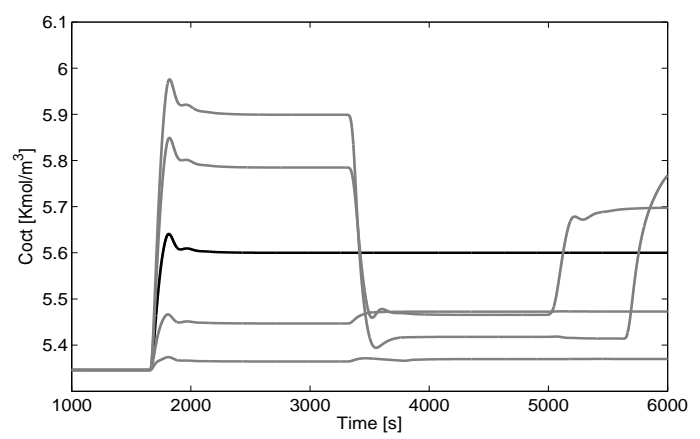

Figure 20: 2-Ethyl-hexanol concentration evolution for a step variation on input $2-$ nominal case vs. uncertain case (PID).
In order to choose the control algorithm with the best performances, the same simulation scenario is considered for the IMC controller. To this end, the closed loop simulations using the IMC controller in Smith Predictor structure under nominal parameter values (solid black line), as well as the simulations considering the variation of the uncertain parameters of the linear plant (solid grey lines), for a reference step variation of the output temperature are presented in Figure 21 and Figure 22.

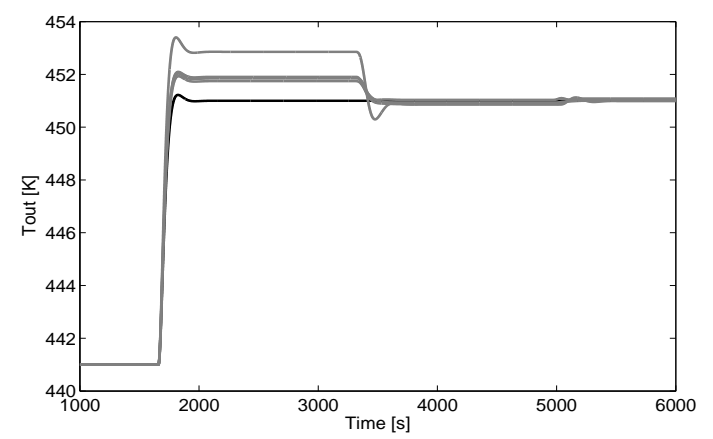

Figure 21: Output temperature evolution for a step variation on input 1 - nominal case vs. uncertain case (IMC).

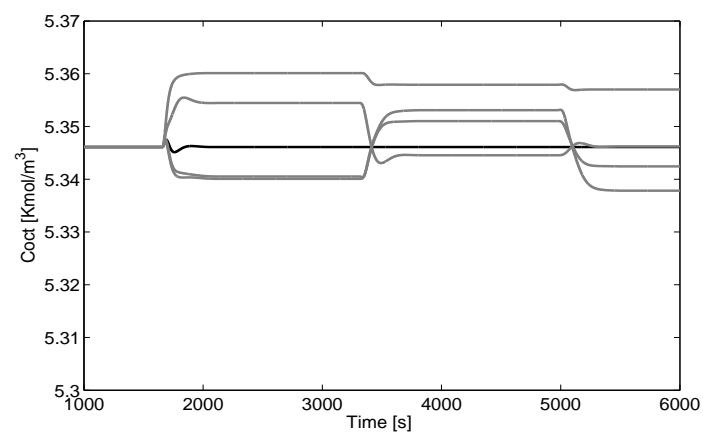

Figure 22: 2-Ethyl-hexanol concentration evolution for a step variation on input 1 - nominal case vs. uncertain case (IMC).

The closed loop simulations using the IMC controller in Smith Predictor structure under nominal parameter values (solid black line), as well as the simulations considering the variation of the uncertain parameters of the linear plant (solid grey lines), for a reference step variation of the 2-ethyl-hexanol concentration are presented in Figure 23 and Figure 24.

Based on the simulation results presented, it can be concluded that the most appropriate control strategy with satisfactory results is the IMC control strategy in Smith Predictor structure.

It can be observed that the differences between the performances of the nominal and perturbed sys- 
tems are smaller than in the case of classical control, Figure 17 to Figure 24. It must be mentioned that the simulation scenarios are considered for four extreme points of operation, implying a very large range of parameter variation. If the variation range of the uncertain parameters is smaller, in normal operation conditions, the differences between the performances of the nominal and perturbed systems are very small.

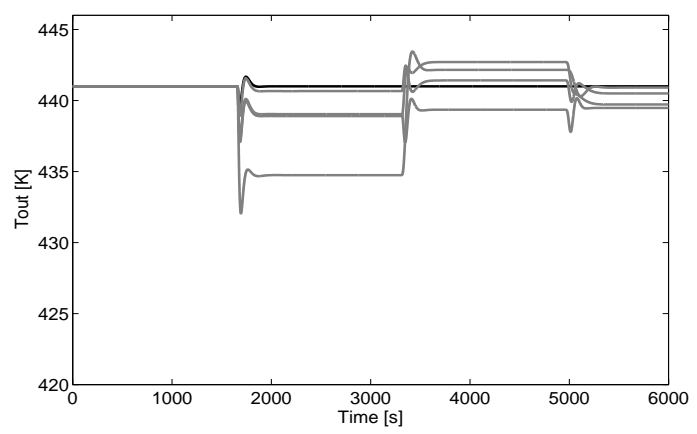

Figure 23: Output temperature evolution for a step variation on input $2-$ nominal case vs. uncertain case (IMC).

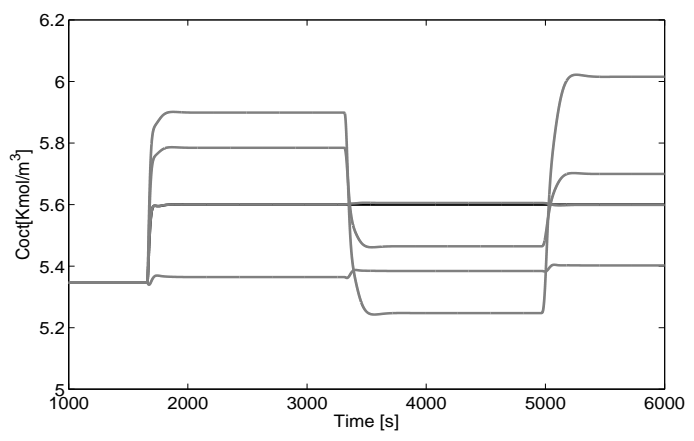

Figure 24: 2-ethyl-hexanol concentration evolution for a step variation on input 2- nominal case vs. uncertain case (IMC).

\section{CONCLUSIONS}

This paper describes the two control strategies of an important chemical process, the 2-ethyl-hexenal hydrogenation process, in a catalytic fixed-bed reactor. This process is a nonlinear system with distributed parameters. However, it is possible to describe the processes that occur inside the reactor by a linear nominal transfer matrix.

Several simulation scenarios are considered in order to choose the control algorithm with the appropriate performances. The simulation results presented in the sixth section of the paper show that the
IMC control presents better performances for setpoint tracking and also for disturbance rejection tests, and better robustness, so it is recommended for implementation. Additionally, this method reduces the design burden of the controllers.

\section{NOMENCLATURE}

$\mathrm{C}_{\mathrm{i}} \quad$ molar concentration $\left(\mathrm{kmol} / \mathrm{m}^{3}\right)$

$\mathrm{c}_{\mathrm{pi}} \quad$ specific heat capacity $(\mathrm{kJ} / \mathrm{kmol} \mathrm{K})$

F volumetric flow rate $\left(\mathrm{m}^{3} / \mathrm{s}\right)$

$\mathrm{T} \quad$ Temperature $(\mathrm{K})$

$\Delta_{\mathrm{R}} \mathrm{H} \quad$ reaction heat $(\mathrm{kJ} / \mathrm{kmol})$

$\mathrm{K}$ adsorption equilibrium constant, component $\mathrm{i}\left(\mathrm{m}^{3} / \mathrm{mol}\right)$

$\mathrm{k}$ rate constant of surface reaction $\mathrm{j}(\mathrm{mol} / \mathrm{s} \mathrm{kg})$

$\mathrm{H}_{\mathrm{H} 2} \quad$ Henry's law constant

$\mathrm{V} \quad$ velocity $(\mathrm{m} / \mathrm{s})$

$\mathrm{S}$ reactor cross-sectional area $\left(\mathrm{m}^{2}\right)$

M molecular mass $(\mathrm{kg} / \mathrm{kmol})$

$\mathrm{a}_{\mathrm{v}} \quad$ specific gas-liquid contact area $\left(\mathrm{m}^{-1}\right)$

$\mathrm{r}$ rate of surface reaction $(\mathrm{mol} / \mathrm{s} \mathrm{kg})$

$\mathrm{t}$ time (s)

$\mathrm{z} \quad$ axial reactor coordinate

Greek Letters

$\rho \quad$ density $\left(\mathrm{kg} / \mathrm{m}^{3}\right)$

\section{Subscripts/Superscripts}

$\mathrm{G}$ gas phase

L liquid phase

i $\quad \mathrm{H}_{2}, 2$-ethyl-hexenal, 2-ethyl-hexanal, 2-ethyl-hexanol

j 2-ethyl-hexenal hydrogenation reaction, 2-ethyl-hexanal hydrogenation reactor

\section{ACKNOWLEDGEMENTS}

This work was supported by a grant of the Romanian National Authority for Scientific Research, CNDI- UEFISCDI, project number 155/2012 PN-IIPT-PCCA-2011-3.2-0591.

\section{REFERENCES}

Attou, A., Boyer, C., Ferschneider, G., Modeling of hydrodynamics of the cocurrent gas-liquid trickle flow in a trickle-bed reactor. Chemical Engineering Science, 54, 785-802 (1999). 
Both, R., Eva-Henrietta Dulf, Clement Feştilă, Robust control of a catalytic 2-ethyl-hexenal hydrogenation reactor. Journal of Chemical Engineering Science, ISI, (2012). doi: 10.1016/j.ces.2012.02. 033.

Both, R., Ana Maria Cormoş, Paul Şerban Agachi, Clement Feştilă, Dynamic modeling and validation of the 2-ethyl-hexenal hydrogenation process. Computer \& Chemical Engineering, 52, 100-111 (2013). http://dx.doi.org/10.1016/j.compchemeng. 2012.11.012

Bozga, G., Muntean, O., Chemical reactors. Bucuresti, Technique Editure, v. 2, Chapter 6 (2001). (In Romanian).

Bristol, E. H., On a new measure of interactions for multivariable process control. IEEE Transactions on Automatic Control, 133-134, 11:1 (1966).

Burghardt, A., Grazyna, B., Miczylaw, J., Kolodziej, A., Hydrodynamics and mass transfer in a threephase fixed bed reactor with cocurrent gas-liquid downflow. The Chemical Engineering Journal, 28, 83-99 (1995).

Chen, J., He, Z.-F. Qi, X., A new control method for MIMO first order time delay non-square systems. Journal of Process Control, 21(4), 538-546 (2011).

Collins, D. J., Grimes, D. E., Davis, B. H., Kinetics of the catalytic hydrogenation 2-ethyl- hexenal. The Canadian Journal of Chemical Engineering, 61, 36-39 (2009).

Coker, K., Modeling of Chemical Kinetic and Reactor Design. Gulf Publishind Company, Chapters 2, 3, 4, 6 (2001).

Gaspar, J., Cormos, A. M., Dynamic modeling and validation of absorber and desorber columns for post-combustion $\mathrm{CO}_{2}$ capture. Computers and Chemical Engineering, 35(10), 2044-2052, (2010). doi:10.1016/j.compchemeng.2010.10.001

Iancu, M., Agachi, S., Optimal process control of an industrial heat integrated fluid catalytic cracking plant using model predictive control. 20th European Symposium on Computer Aided Process Engineering ESCAPE (2010).

ICIS, <http://www.icis.com/> (Accessed: April 04, 2011).

Iliuta, I., Thyrion, F. C., Bolle, L., Giot, M., Comparison of hydrodynamic parameters for countercurrent and cocurrent flow through packed beds. Chemical Engineering Science, 20, 171-181 (1997).

Kvamsdal, H. M., Jakobsen, J. P., Hoff, K. A., Dynamic modeling and simulation of a $\mathrm{CO}_{2}$ absorber column for postcombustion $\mathrm{CO}_{2}$ capture. Chemical Engineering Process, 48, 135-144 (2009).
Lawal, A., Wang, M., Stephenson, P., Koumpouras, G., Yeung, H., Dynamic modeling and analysis of post-combustion $\mathrm{CO}_{2}$ chemical adsorption process for coal-fired power plants. Fuel, 89, 27912801 (2010).

McAvoy, T. J., Some results on dynamic interaction analysis of complex control systems. Ind. Eng. Chem. Process Des. Dev., 22, 42 (1983).

Mahmud, M., Faizi, A. K., Anchoori, V., Al-Qahtani, A., Liquid phase catalytic hydrogenation process to convert aldehydes to the corresponding alcohols. Patent No. US 6600078 B1 (2003).

MATLAB Users Guide. The MathWorks Inc (2008).

Mattos, A. R. J. M., Probst, S. H., Afonso, J. C., Schmal, M., Hydrogenation of 2-ethyl-hexen-2-al on $\mathrm{Ni} / \mathrm{Al}_{2} \mathrm{O}_{3}$ Catalyst. Journal of Brazilian Chemical Society, 15, 760-766 (2004).

Melo, D. N. C., Costa, C. B. B., Toledo, E. C. V., Santos, M. M., Maciel, M. R. W., Maciel Filho. R., Evaluation of control algorithms for three phase hydrogenation catalytic reactors. Chemical Engineering Journal, 141, 250-263 (2008).

National Institute of Standards and Technology, $<$ http://www.nist.gov/> (Accessed: April 04, 2011).

Niklasson, C., Kinetics of adsorption and reaction for consecutive hydrogenation of 2-ethylhexenal on $\mathrm{Ni} / \mathrm{SiO}_{2}$ catalyst. Industrial \& Engineering Chemistry Research, 26, 403-410 (1987).

Niklasson, C., Kinetics of hydrogenation of 2-ethylhexenal and hydrogen/deuterium exchange on a palladium/silica catalyst in a continuously stirred tank reactor. Industrial \& Engineering Chemistry Research, 27(11), 1990-1995 (1988).

Olah, G. A., Molnar, A., Hydrocarbon Chemistry. 2nd Ed., New York, John Wiley \& Sons, Chapter 11 (2003).

Pavlov, C. F., Romankov, P. G., Processes and Apparatus in Chemical Engineering. Bucuresti, Technique Editure (1981). (In Romanian).

Perry, R. H., Green, D. W., Perry's Chemical Engineers' Handbook. 8th Ed., New York, McGraw-Hill (1999).

Pop, C. I., De Keyser, R., Ionescu. C., A simplified control method for multivariable stable nonsquare systems with multiple time delays. Control \& Automation (MED), 19th Mediterranean Conference, Proceedings, 382-387 (2011). doi: 10.1109/MED. 2011.5983051.

Silva, J. D, Lima, F. R. A., Abreu, C. A. M., Knoechelmann, A., Experimental analysis and evaluation of the mass transfer process in a trickle-bed reactor. Brazilian Journal of Chemical Engineering, 20, 375-390 (2003).

Simpson, T. W., Peplinski, J. D., Koch, P. N., Allen, 
J. K., Metamodels for computer-based engineering design: Survey and recommendations. Engineering with Computers, 17(2), 129-150 (2001).

Skogestad, S., Postlethwaite, I., Multivariable Feedback Control: Analysis and Design. New York, John Wiley \& Sons (2005).

Smelder, G., Kinetic Analysis of the liquid phase hydrogenation of 2-ethyl-hexenal in the presence of supported Ni,Pd and Ni-S catalyst. The Canadian Journal of Chemical Engineering, 67, 51-61 (1989).

SRI Consulting, IHS Inc, < http://www. sriconsulting. com/> (Accessed: March 07, 2011).

Tobiesen, F. A., Juliussen, O., Svendsen, H. F., Experimental validation of a rigorous desorber model for $\mathrm{CO}_{2}$ postcombustion capture. Chemical Engineering Science, 63, 2641-2656 (2008).
Wang, Q. G., Ye, Z., Cai, W-J., Hang, C-C., PID Control for Multivariable Processes. SpringerVerlag Berlin Heidelberg (2008).

Wang, Q. G., Zou, B., Zhang, Y., Decoupling Smith predictor design for multivariable systems with multiple time delays. Chemical Engineering Research and Design, 78, 565-572 (2000).

Wang, Q. G., Zhang, Y., M. Chiu, S., Decoupling internal model control for multivariable systems with multiple time delays. Chemical Engineering Science, 57, 115-124 (2002).

World Resources Institute, <http://www.wri.org/> (Accessed: November 23, 2009).

Xiong, Q., Cai, W. and He, M., A practical loop pairing criterion for multivariable processes. Journal of Process Control, 15, 741-747 (2005). 